\title{
4D printing of stretchable nanocookie@conduit material hosting biocues and magnetoelectric stimulation for neurite sprouting
}

\author{
Jen-Hung Fang ${ }^{1}$, Hao-Hsiang Hsu' ${ }^{1}$ Ru-Siou Hsu' ${ }^{1}$, Chih-Kang Peng ${ }^{1}$, Yu-Jen Lu², You-Yin Chen ${ }^{3}$, \\ San-Yuan Chen (10 ${ }^{4,5,6,7}$ and Shang-Hsiu Hu (10 ${ }^{1}$
}

\begin{abstract}
A high-frequency magnetic field (MF) generates an electric current by charging conductors that enable the induction of various biological processes, including changes in cell fate and programming. In this study, we show that electromagnetized carbon porous nanocookies (NCs) under MF treatment facilitate magnetoelectric conversion for growth factor release and cell stimulation to induce neuron cell differentiation and proliferation in vitro and in vivo. Integrating four-dimensional printing technology, the NCs are exposed on the surface, which enhances the cell adhesion and allows direct manipulation of electromagnetic stimulation of the cells. Remarkably, large amounts of growth factor encapsulated in NC@conduit resulted in excellent permeability and on-demand release, improving the in vivo layers of myelin sheaths and directing the axon orientation at 1 month postimplantation. This study offers proof of principle for MFguided in vivo neuron regeneration as a potentially viable tissue regeneration approach for neuronal diseases.
\end{abstract}

\section{Introduction}

Four-dimensional (4D) printed soft materials from the molecular design of a three-dimensional (3D) printed object capable of transforming intrinsic properties over time in response to environmental stimuli, such as voltage, temperature, and chemical reaction, are of considerable interest in soft microrobotics, computational folding, and memory materials ${ }^{1-5}$. Printed complex geometries offering personal biomedical engineering applications are required in new drug delivery formulations ${ }^{6}$, tumor therapy ${ }^{7,8}$, living devices ${ }^{9}$, and actuators ${ }^{10,11}$. For example, 3D printed tablets or oral compartmental dosage forms exhibit a controllable-by-design delivery system to program drug release patterns ${ }^{12}$. In the regeneration of volumetric tissue, 3D cell printing scaffolds release cell or

\footnotetext{
Correspondence: San-Yuan Chen (sanyuanchen@mail.nctu.edu.tw) or ShangHsiuHu (shhu@mx.nthu.edu.tw)

'Department of Biomedical Engineering and Environmental Sciences, National Tsing Hua University, Hsinchu 300, Taiwan

${ }^{2}$ Center for Biomedical Engineering, Chang Gung University, Taoyuan City 333, Taiwan

Full list of author information is available at the end of the article
}

growth factors to stimulate blood vessel reconstruction and manipulate stem cell differentiation ${ }^{13}$. However, only a few 3D printing techniques can give a uniquely controllable surface roughness and on-demand electric current in a biomicroenvironment to induce various biological processes. Through modifying this technology, a versatile structure can be potentially optimized with the material design in one compact system to facilitate personalized medicine.

Even with the current breakthroughs in 3D printing, the challenges of printed tissue-regenerated materials still exist in on-demand biocues and electrical stimulus for programming neuron-related cells, which is a key factor to promote neurite sprouting and Schwann cell (SC) migration across the injury site ${ }^{14,15}$, especially for peripheral nerve injury $(\mathrm{PNI})^{16,17}$. In addition, the electric current enables the induction of different biological processes, including changes in cell fate and programming ${ }^{18}$. However, deep nerve stimulus via electric current usually requires invasive implantation of an electrode in the tissue $^{19}$. To address this issue, by combination with a

\section{(c) The Author(s) 2020}

(c) Open Access This article is licensed under a Creative Commons Attribution 4.0 International License, which permits use, sharing, adaptation, distribution and reproduction cc) in any medium or format, as long as you give appropriate credit to the original author(s) and the source, provide a link to the Creative Commons license, and indicate if changes were made. The images or other third party material in this article are included in the article's Creative Commons license, unless indicated otherwise in a credit line to the material. If material is not included in the article's Creative Commons license and your intended use is not permitted by statutory regulation or exceeds the permitted use, you will need to obtain permission directly from the copyright holder. To view a copy of this license, visit http://creativecommons.org/licenses/by/4.0/. 
nanoconductor, a high-frequency magnetic field (MF) can remotely induce electric currents (eddy currents) by charging objects (e.g., gold and graphene-based materials). Such physical energy has also been capable of controlling drug release or promoting osteogenesis and neurogenesis for cell proliferation ${ }^{20,21}$. Regarding neuron stimuli, Yoo and coworkers demonstrated that electromagnetized gold nanoparticles could facilitate the lineage reprogramming of dopamine neurons ${ }^{22}$. Nevertheless, these nanoconductors have to contact the cell directly, which is difficult to achieve by traditional hydrogel modeling since the particles are embedded in the gel rather than exposed on the surface. In contrast, 3D printing by light curing can expose the nanoconductors on the surface of the matrix and be integrated with an MF to stimulate cells due to the exquisite curing process, which inherently creates a broad strategy to affect tissue regeneration.

To amplify the functions of 3D printing, a simple approach is to integrate nanoconductors into printed soft matter. Graphene exhibiting biocompatibility and conductivity has been utilized in drug delivery ${ }^{23}$, stem cell differentiation $^{24}$, sensors $^{25,26}$, and tissue engineering ${ }^{27}$. By incorporating this material, 3D printing of graphene elastomers with excellent electrical, flexible, and neurogenic properties can expose graphene on the surface, and support of neurogenic differentiation ${ }^{28}$. Furthermore, Hsu et al. used 3D printed polyurethane (PU)-based hydrogel scaffolds with bioactive ingredients, which induced chondrogenic differentiation of mesenchymal stem cells for cartilage regeneration ${ }^{29}$. A commercial product, Opsite ${ }^{\circledR}$, that was applied as a transparent adhesive PUbased dressing exhibited oxygen permeability and accelerated dermal repair ${ }^{30}$. Such PU-based scaffolds are beneficial to injured nerve tissue with long-term supports, including rigidity, topographic cues, and nutrient permeability $^{31-34}$. These developments of 3D printed biocompatible elastomers offer potential opportunities for tissue engineering.

Here, we developed a 3D printing method to prepare a stretchable and biocompatible conduit with a nanocomposite composed of reduced graphene oxide (GO) nanosheets, nanocookies (NCs), for peripheral nerve regeneration. NCs with mesoporous silica exposed on the surface of the conduits provide magnetoelectric conversion, protein adsorption, and cell stimulation. The internal microchannels inside were simply and simultaneously prepared by an additive manufacturing process, in contrast to earlier studies ${ }^{34}$. On-demand neuron growth factor (NGF) release and cell stimulus were achieved in vitro under MF treatment to effectively induce cell proliferation and differentiation, and the implants promoted axon outgrowth after a peripheral nerve was transected $10 \mathrm{~mm}$ from the bifurcation point of the sciatic nerve of Sprague-Dawley (SD) rats.

\section{Materials and methods}

\section{Synthesis of GO nanosheets and NCs}

GO was synthesized via a modified Hummers method ${ }^{35}$. First, graphite powder $(3.0 \mathrm{~g})$ was mixed with concentrated $\mathrm{H}_{2} \mathrm{SO}_{4}(69 \mathrm{ml})$ and $\mathrm{NaNO}_{3}(1.5 \mathrm{~g})$. After $5 \mathrm{~min}$, the mixture was cooled in an ice bath to $0{ }^{\circ} \mathrm{C}$. Then, $\mathrm{KMnO}_{4}(9.0 \mathrm{~g})$ was added slowly because the reaction temperature had to be kept $<20^{\circ} \mathrm{C}$. Then, the mixture was stirred and heated at $35^{\circ} \mathrm{C}$. After $12 \mathrm{~h}, 30 \% \mathrm{H}_{2} \mathrm{O}_{2}$ was added to the mixture until the solution turned bright yellow. The mixture was washed by adding $30 \% \mathrm{HCl}(200 \mathrm{ml})$ and centrifuged at 6000 r.p.m. for $10 \mathrm{~min}$ two times. The precipitates were collected and washed with deionized water until the $\mathrm{pH}$ of the washings became neutral. Finally, after drying, GO was collected. The synthesis of NCs was previously described ${ }^{36}$. Briefly, $0.1 \mathrm{~g}$ of cetyl trimethyl ammonium bromide (CTAB) and $0.4 \mathrm{mg}$ GO were placed in $30 \mathrm{ml}$ of deionized water, and the mixture was stirred at $60{ }^{\circ} \mathrm{C}$ for $30 \mathrm{~min}$. Then, $14.4 \mathrm{ml}$ of octane, $2.45 \mathrm{ml}$ of styrene, $0.022 \mathrm{~g}$ of L-lysine, and $1.071 \mathrm{ml}$ of TEOS were added into the mixture. After the mixture was mixed, $0.0371 \mathrm{~g}$ of AIBA was immediately added, and the solution was stirred at $60^{\circ} \mathrm{C}$ for $3 \mathrm{~h}$. Next, the heating stopped, and the mixture was cooled naturally to room temperature $(\sim 12 \mathrm{~h})$. The mixture was washed with deionized water three times and then centrifuged at 6000 r.p.m. for $10 \mathrm{~min}$. The gray powder was collected by drying under vacuum at $60^{\circ} \mathrm{C}$. Finally, the gray powder was heated at $450^{\circ} \mathrm{C}$ for $3 \mathrm{~h}$ under $\mathrm{N}_{2}$ gas and washed with deionized water three times to remove impurities. The material was finally dried to a powder and termed NCs.

\section{Preparation of 3D printed conduit}

A digital light processing (DLP) 3D printer was purchased from MiiCraft (MiiCraft plus, Taiwan), which was controlled by a computer via an RJ-45 cable. The maximum build area was $43 \mathrm{~mm} \times 27 \mathrm{~m} \times 180 \mathrm{~m}$, and the minimum thickness of the product was $5 \mu \mathrm{m}$, which was adjustable up to $200 \mu \mathrm{m}$. An LED projector with a wavelength of $405 \mathrm{~nm}$ at a power of $12.5 \mathrm{~mW}$ was equipped in the printer. 3D printed resin was prepared by some types of light-curable molecules, including $17.8 \mathrm{~g}$ of 4-hydroxybutyl acrylate (4-HBA), $2 \mathrm{~g}$ of urethanepolyethylene glycol-polypropylene glycol (PU-EO-PO) monomer, and $0.2 \mathrm{~g}$ of Irgacure 819 as the initiator, which were well mixed and protected from light at room temperature for $3 \mathrm{~h}$. Resins with $0.1 \%$ NCs were prepared by mixing $17.6 \mathrm{~g}$ of 4 -HBA, $2 \mathrm{~g}$ of PU-EO-PO monomer, $0.2 \mathrm{~g}$ of Irgacure 819 , and $0.02 \mathrm{~g}$ of $\mathrm{NCs}$; resins containing $1 \%$ NCs were prepared by mixing $17.6 \mathrm{~g}$ of 4-HBA, $2 \mathrm{~g}$ of $230 \mathrm{TH}, 0.2 \mathrm{~g}$ of Irgacure 819 , and $0.2 \mathrm{~g}$ of NCs. The resin underwent ultrasonication to avoid bubbles and precipitation. The 3D objects were drawn by 3D computeraided design (CAD), and were then imported to slicer software (MiiUtility3.2) and uploaded to the 3D printer. 
The printing thickness per layer was $100 \mu \mathrm{m}$, and the curing time was $10 \mathrm{~s}$ per layer. After filling the printing pool with resin, the stage sank into the resin $100 \mu \mathrm{m}$ from the bottom of the pool. The structure was polymerized and formed layer by layer on the stage. The 3D customized nerve guides were finally cured with further irradiation (postcuring under $18 \mathrm{~W}$ UVA lamp irradiation) at room temperature followed by washing with deionized water twice, and sterilization via an autoclave at a temperature of $121^{\circ} \mathrm{C}$ at $15 \mathrm{psi}$ for further in vitro and in vivo experiments.

\section{Characterizations of NCs and the 3D printed conduit}

The structure of the NCs was revealed by transmission electron microscopy (TEM, JEM-2100, Japan). The NCs were well dispersed in deionized water and dried on carbon film-supported copper grids under vacuum at room temperature for specimen preparation. Raman spectroscopy was used to determine the nonpolar bonds of graphite, GO, and NCs. All powders were dried on a silicon wafer and measured with a UniNanoTech system (UniRAM, South Korea) at $1.58 \mathrm{eV}$ by excitation with a $514 \mathrm{~nm}$ laser. Dynamic light scattering (DLS) analysis by using a particle sizer (Nano-ZS, Malvern, UK) determined the average size, and size distribution of GO and NCs diluted with deionized water. $\mathrm{N}_{2}$ adsorption-desorption isotherms were measured by using an Autosorb 1 MP system (Quantachrome Instruments, Florida, USA). The Barrett-Joyner-Halenda $(\mathrm{BJH})$ and Brunauer-Emmett-Teller (BET) methods were used to calculate the pore size and surface area of the samples, respectively. Graphite, GO, and NCs were dried at $70{ }^{\circ} \mathrm{C}$ under vacuum for $24 \mathrm{~h}$, and degassed at $200{ }^{\circ} \mathrm{C}$ for $2 \mathrm{~h}$ before analysis. Field-dependent magnetization curves were evaluated by a superconducting quantum interference device (SQUID, Quantum Design MPMS-XL7, USA) from $-40,000$ to $40,000 \mathrm{Oe}$ at $6 \mathrm{~K}$ and $300 \mathrm{~K}$. The field-sensitive temperature dependence of magnetization was evaluated from $5 \mathrm{~K}$ to $300 \mathrm{~K}$ at $50 \mathrm{Oe}$. Atomic configurations and the surface elemental composition were examined by X-ray photoelectron spectroscopy (XPS, PHI Quantera II). The morphologies of the 3D printed conduit were determined by field emission scanning electron microscopy (FE-SEM, JSM-7000F, Japan). The samples were anchored to SEM specimen mounts using double-sided carbon tape and sputter deposited with platinum at $10 \mathrm{~mA}$ for $120 \mathrm{~s}$. We followed ASTM-D638-14 to print the dog-bone shape for tensile testing (4468, Instron, USA) with a $1 \mathrm{kN}$ load cell under a constant strain rate of $10 \mathrm{~mm}$ per min. Prior to all tests, the load was reset, and the load cell was calibrated. The printed samples were then postured for 0,1 , and $4 \mathrm{~h}$ to evaluate the stress and strain diagram $(n=6)$. The slope of the linear region from the stress-strain curve was calculated as Young's modulus. The ultimate tensile strength was taken as the maximum stress reached before failure.

\section{Cell culture and in vitro biocompatibility of 3D printed matrix}

HIG-82 cells were cultured in Ham's F12 medium containing $10 \% \quad(\mathrm{v} / \mathrm{v}) \quad$ FBS and $1 \% \quad(\mathrm{v} / \mathrm{v})$ penicillin-streptomycin. Neuro-2a (N2a) cells were cultured in EMEM containing 10\% (v/v) FBS and 1\% (v/v) penicillin-streptomycin. SCs were cultured in DMEM containing $0.094 \mathrm{gl}^{-1} \mathrm{D}$-valine, 10\% (v/v) FBS, 1\% (v/v) $\mathrm{N} 2$ supplement, $20 \mu \mathrm{g} \mathrm{ml}^{-1}$ (wt/v) bovine pituitary extract, $5 \mu \mathrm{M}$ forskolin, and $1 \%(\mathrm{v} / \mathrm{v})$ penicillin/streptomycin. PC12 cells were cultured in RPMI-1640 with $10 \%(\mathrm{v} / \mathrm{v}) \mathrm{HS}$, $5 \%(\mathrm{v} / \mathrm{v})$ FBS, and $1 \%(\mathrm{v} / \mathrm{v})$ penicillin-streptomycin. The cells were cultured in complete medium at $37^{\circ} \mathrm{C}$ in a humidified atmosphere of $5 \%$ carbon dioxide in air. The medium was changed every other day. The cytotoxicity followed the tests for in vitro cytotoxicity (ISO 10993-5). Briefly, we prepared samples in $1 \times 1 \times 0.1 \mathrm{~cm}^{3}$ by 3D printing and then washed them with deionized water, as well as ethanol three times. The samples were sterilized via an autoclave at a temperature of $121^{\circ} \mathrm{C}$ at $15 \mathrm{psi}$ for $30 \mathrm{~min}$ and extracted in culture medium with serum following ISO 10993-12. After seeding cells in 96-well plates (10,000 cells per well) for 1 day, we added the extraction into each well for another 1-day incubation. Finally, we used the MTT agent to detect toxicity at wavelengths of $570 \mathrm{~nm}$ and $650 \mathrm{~nm}$ as a reference. Cell viability was calculated by comparison with untreated cells and calculated according to the following: cell viability $(\%)=$ absorbance of experimental group/absorbance of control group. For the external MF test, we applied an MF above the cell culture dish $1 \mathrm{~cm}$ away at a power of $3.2 \mathrm{~kW}$ and frequency of $1 \mathrm{MHz}$ for $15 \mathrm{~min}$ before using the MTT agent.

For observing cells on the 3D printed matrix by electron microscopy, cells that grew on the 3D printed matrix were further fixed with $2.5 \%$ glutaraldehyde for $30 \mathrm{~min}$ and then $2 \%$ osmium tetroxide $\left(\mathrm{OsO}_{4}\right)$ in PBS. Cells were then washed three times with PBS for 10 min each followed by dehydration through a graded series of ethanol sequentially at $10,20,30,50$, and $70 \%$ (30 min for each step), and immersed in $100 \%$ acetone for 30 min each.

\section{Flow cytometry and the effect of NCs on PC12 cells}

QDs were adopted to label NCs for tracking purposes in in vitro experiments. The QDs were encapsulated in NCs by first mixing $2.5 \mathrm{mg}$ of QDs and $10 \mathrm{mg}$ of NCs dissolved in chloroform solution, stirring for $24 \mathrm{~h}$. Then, the samples, NCs/QDs, were dried under vacuum at $60^{\circ} \mathrm{C}$ for $1 \mathrm{~h}$. To remove free QDs, the samples were washed with deionized water three times and collected by centrifugation at 6000 r.p.m. for $8 \mathrm{~min}$. Afterward, 500,000 PC12 cells were cultured in six-well plates with $1 \mathrm{ml}$ of medium for $24 \mathrm{~h}$, and then incubated with NCs/QDs for $0.5,1$, and $4 \mathrm{~h}$. Next, the cells were washed with PBS and soaked in trypsin-EDTA. After harvesting the cells with the culture 
medium, the cells were centrifuged at 500 r.p.m. for $5 \mathrm{~min}$. Finally, the cells were rinsed with PBS and collected for flow cytometry analysis. In addition, 10,000 PC12 cells were seeded and grown on glass coverslips in wells at $37^{\circ} \mathrm{C}$ for $24 \mathrm{~h}$ in the incubator, and then treated with NCs/QDs for $4 \mathrm{~h}$. After incubation, cells were stimulated by MF (power cube 32/900, President Honor Industries, Taiwan) at a power of $3.2 \mathrm{~kW}$ and frequency of $1 \mathrm{MHz}$ for $15 \mathrm{~min}$. The cells were then continuously incubated for 3 days. The medium was removed, and the cells were washed twice with PBS for the dye procedure. We used $3.7 \%$ formaldehyde (PBS solution) to fix the cells for $20 \mathrm{~min}$ at $37^{\circ} \mathrm{C}$, and then washed the wells twice with PBS. Next, we permeabilized the cells by immersion in $0.1 \%$ Triton X-100 (diluted in PBS solution) for $20 \mathrm{~min}$ at $37^{\circ} \mathrm{C}$, and then washed the wells twice with PBS. Finally, we stained the nucleus and phalloidin by using DAPI $\left(1 \mu \mathrm{g} \mathrm{ml}^{-1}\right)$ and F-actin $\left(1 \mathrm{unit} \mathrm{ml}^{-1}\right)$ for $30 \mathrm{~min}$, respectively. The images were obtained on a Zeiss LSM 780 CLSM equipped with an argon-ion laser.

\section{Cell behaviors with physical cues and PC12 cell differentiation}

3D printed conduits with and without microchannels were washed with both deionized water and ethanol three times. Then, the conduits were sterilized via an autoclave at a temperature of $121^{\circ} \mathrm{C}$ at $15 \mathrm{psi}$ for $30 \mathrm{~min}$. Sterilized 3D printed planes were placed in six-well plates. Next, 10,000 HIG-82 cells with culture medium were seeded and cultivated on the $3 \mathrm{D}$ printed matrix for $1-4$ days at $37^{\circ} \mathrm{C}$, and the cultures were then imaged, where DAPI stained the nucleus and phalloidin stained F-actin. The images were obtained on a Zeiss LSM 780 CLSM equipped with an argon-ion laser. The binary two-dimensional (2D) images were then processed by Nikon NIS-elements software, providing a basis for the printed physical cue orientation at $0^{\circ}$. The orientation was calculated by the angle at Feret's maximum diameter. The resultant power spectra were then generated by plotting a Gaussian distribution fitting along a circumferential profile. For PC12 cell differentiation, 10,000 PC12 cells were seeded and grown on glass coverslips in the wells. The medium was changed to RPMI-1640 with $2 \%$ (v/v) HS, 1\% (v/v) FBS, $1 \%$ $(\mathrm{v} / \mathrm{v})$ penicillin-streptomycin, and $100 \mathrm{ng} \mathrm{ml}^{-1} \mathrm{NGF}$ in the control group for 5 days at $37^{\circ} \mathrm{C}$. Sterilized 3D printed planes were immersed in $100 \mathrm{ng} \mathrm{ml}^{-1}$ NGF for 2 days and then placed in six-well plates. Next, 10,000 PC12 cells were seeded and grown on the 3D printed planes with RPMI-1640 with $2 \% \quad(\mathrm{v} / \mathrm{v}) \quad \mathrm{HS}, \quad 1 \% \quad(\mathrm{v} / \mathrm{v}) \quad$ FBS, and $1 \% \quad(\mathrm{v} / \mathrm{v})$ penicillin-streptomycin at $37^{\circ} \mathrm{C}$ for 1 day. Then, cells were stimulated by MF treatment above the cell culture dish $1 \mathrm{~cm}$ away at a power of $3.2 \mathrm{~kW}$ and frequency of $1 \mathrm{MHz}$ for $15 \mathrm{~min}$. The cells were then continuously incubated for 4 days. The medium was finally removed, and the cells were washed twice with PBS for the dye procedure. We used 3.7\% formaldehyde (PBS solution) to fix the cells for $20 \mathrm{~min}$ at $37^{\circ} \mathrm{C}$, and then washed the wells twice with PBS. Next, we permeabilized the cells by immersion in $0.1 \%$ Triton X-100 (PBS solution) for $20 \mathrm{~min}$ at $37^{\circ} \mathrm{C}$ and then washed the well twice with PBS. Finally, we stained the nucleus and phalloidin by using DAPI $\left(1 \mu \mathrm{g} \mathrm{ml}^{-1}\right)$ and F-actin $\left(1\right.$ unit ml $\left.^{-1}\right)$ for $30 \mathrm{~min}$, respectively. The images were obtained on a Zeiss LSM 780 CLSM.

\section{Animal and surgical procedure}

All SD male rats aged 3 weeks were purchased from BioLASCO Taiwan Co., Ltd. All rats were maintained under conditions at $22 \pm 2{ }^{\circ} \mathrm{C}$ on a $12 \mathrm{~h}$ dark day cycle with access to water and food. All experiments were approved by the guidelines of the Institution of Animal Care and Use Committee of National Tsing Hua University (NTHU). All rats were randomly selected to undergo repair with the left limb by a 3D printed conduit. Rats were fixed on the table, and the sciatic nerve was exposed by as small an incision as possible at the posterior of the left hind near the bifurcation point to allow both observation and the 3D printed conduit to be subcutaneously placed. In the autograft group, the sciatic nerve was cut $10 \mathrm{~mm}$ (gap) and inversely sutured with 9-0 sutures under a microscope, four times on each side. For the conduit group, muscle and skin incisions were closed by layers, and the animals could freely eat food and water after the surgery.

\section{Functional assessment of walking track analysis}

All paws of rats were recorded by a digital single-lens reflex camera postsurgery for 1 month. The feet of the injured limbs were measured with the following parameters: (1) toe spread (TS), the distance between the first and the fifth toes; (2) intermediate toe spread (ITS), the distance between the second and the fourth toes; and (3) Print length (PL), the distance from the heel to the third toe. The sciatic function index (SFI) was calculated ( $\mathrm{N}$ : the contralateral hind limb; $\mathrm{E}$ : the experimental hind limb; a value of -100 implies total impairment $): \quad \mathrm{SFI}=-38.3 \quad(\mathrm{EPL}-\mathrm{NPL}) / \mathrm{NPL}+109.5$ $($ ETS - NTS)/NTS $+13.3($ EITS - NITS)/NITS -8.8 .

\section{Relative gastrocnemius muscle weight}

All rats were sacrificed via inhalation of carbon dioxide postsurgery for 1 month. The regenerated nerve and adjacent nerve tissue were dissected in the container with $4 \%$ PFA (4 wt\%) in $0.1 \mathrm{M} \mathrm{PBS}$ for 1 day. The gastrocnemius muscle of both hind limbs was also excised and weighed to analyze relative gastrocnemius muscle weight, and finally immersed in 4\% PFA (4 wt\%) in $0.1 \mathrm{M}$ PBS for 3 days.

\section{Immunohistochemistry}

The harvested conduit with regenerative nerves was sequentially immersed in 10\% (wt\%) sucrose for $30 \mathrm{~min}$, 

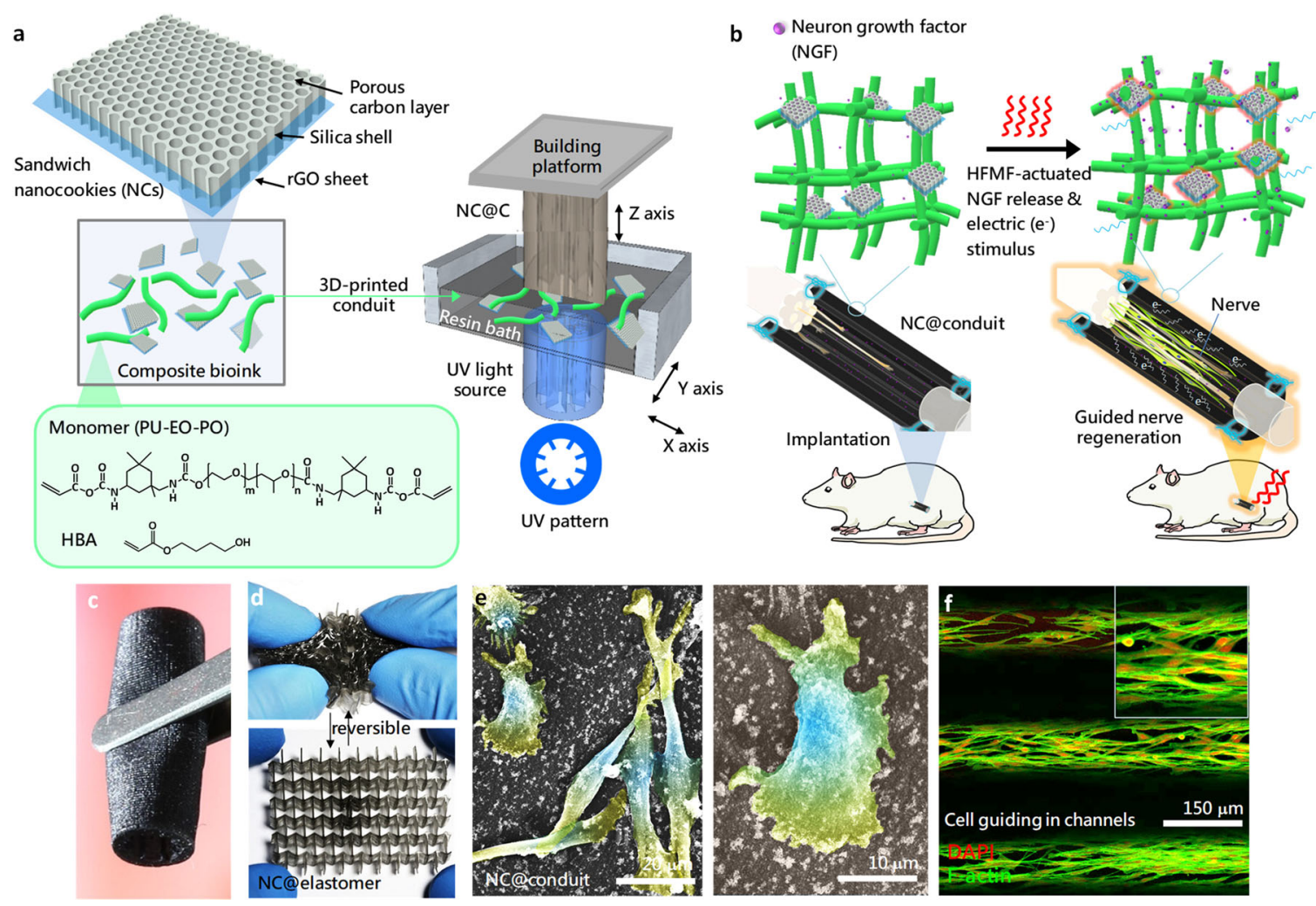

Fig. 1 4D Printing of stretchable NC@C with magnetoelectric conversion capability to release growth factors and simulate cells for neurite sprouting. a Composite bioink composed of 4-HBA, PU-EO-PO monomer, initiator, and NCs made through 3D printing for NC@C fabrication. b NC@C under MF treatment facilitated magnetoelectric conversion for growth factor release and cell stimulation to induce neuron cell differentiation. c Image of a 3D printed NC@C with elastic properties.d The elastic properties of NC@C showed stretchable properties. e SEM image of cells adhered on NC@C. The roughness of the exposed NCs on the surface improved cell attachment.fCLSM images of cells proliferating on NC@C with microchannels. DAPl-stained nuclei (red) and phalloidin-stained F-actin (green).

$15 \%$ (wt\%) sucrose for $30 \mathrm{~min}$, and $30 \%$ (wt\%) sucrose for $30 \mathrm{~min}$ for dehydration. Then, the tissues were embedded in OCT, and horizontal and longitudinal cryostat sections ( $10 \mu \mathrm{m}$ in thickness) were sliced, and stained for immunohistochemical analysis. In brief, the frozen sections were immersed in ethanol at $-20^{\circ} \mathrm{C}$ for $10 \mathrm{~min}$ and washed with $\mathrm{PBS}$ at room temperature three times for $5 \mathrm{~min}$ each. The sections were stained with anti-beta IIItubulin antibody [TU-20] (1:200, rabbit IgG1) for regenerated axons overnight at $4{ }^{\circ} \mathrm{C}$, followed by goat antimouse IgG $(\mathrm{H}+\mathrm{L})$ antibody (FITC) for $2 \mathrm{~h}$ at room temperature. After washing three times with PBS, the sections were stained with mounting medium containing DAPI. Finally, all stained sections were visualized by a ZEISS LSM 780 microscope. The binary 2D images were then processed by Nikon NIS-elements software, providing a basis for the printed physical cue orientation at $0^{\circ}$. The orientation was calculated by the angle at Feret's maximum diameter. The resultant power spectra were then generated by plotting a Gaussian distribution fitting along a circumferential profile.

\section{Stereographic analysis of sciatic nerve regeneration}

The harvested regenerative nerve (at the middle between each endpoint) was fixed with $3 \%$ glutaraldehyde at $4{ }^{\circ} \mathrm{C}$ and then postfixed with $1 \% \mathrm{OsO}_{4}$. The specimens were serially dehydrated with ethanol and embedded in Araldite 502. The specimens were cut into semithin sections followed by staining with $1 \%$ toluidine blue. Ultrathin sections $(60 \mathrm{~nm})$ were lifted onto formvar-coated grids, poststained with lead citrate and uranyl acetate, and subsequently imaged using electron microscopy.

\section{Results and discussion}

Fabrication and characterization of $\mathrm{NC} @ \mathrm{C}$

A composite conduit (NC@conduit, $\mathrm{NC@C)} \mathrm{integrating}$ features of mesoporous carbon sheets and proteinpermeable elastomers to promote peripheral nerve regeneration under magnetoelectric stimulation was developed in this study. As shown in Fig. 1a, a bioink composed of mesoporous carbon layers on a GO sheet (sandwich NCs) and 4-HBA/PU-EO-PO monomer was 


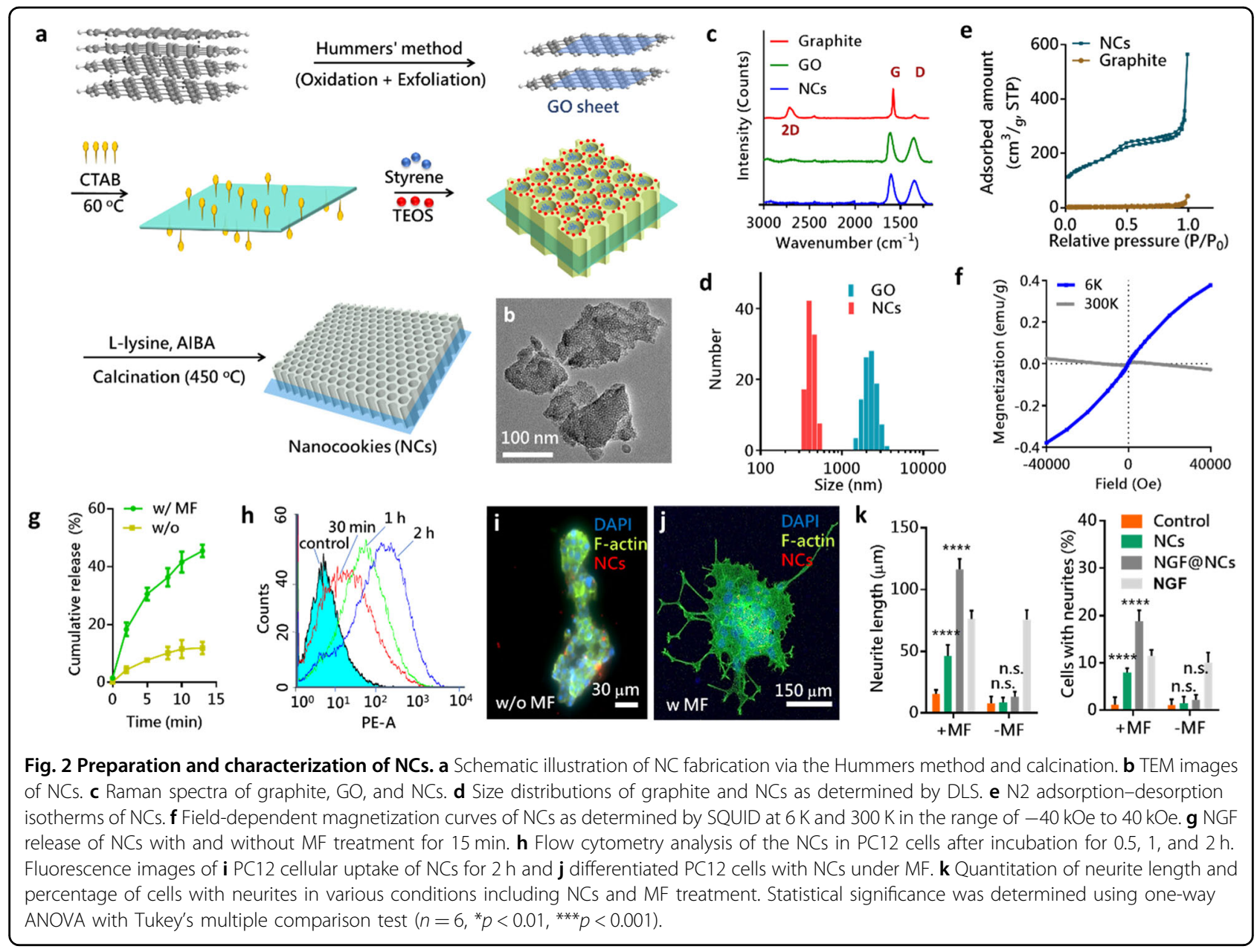

placed in a 3D printer resin bath. Then, UV light possessing a channel pattern in a ring was applied to cure the bioink to form a flexible composite NC@C with internal channels through DLP 3D printing. As shown in Fig. 1b, 3D printed NC@C loaded with a large payload of NGF was implanted to implement the spatial distribution of NGF for the promotion of axon outgrowth after a peripheral nerve was transected $10 \mathrm{~mm}$ from the bifurcation point of the sciatic nerve of SD rats. Upon receiving MF irradiation, the NFG encapsulated in NC@C showed excellent permeability and on-demand release to induce the differentiation and proliferation of nerve cells in vivo, while simultaneously supplying electromagnetic stimulation to cells. Via UV pattern design, conduits with various channels can be prepared and loaded with drugs or proteins in the matrix (Supplementary Fig. S1). After embedding NCs, the printed conduit and composite maintained good elastic and stretchable properties (Fig. 1c, d). A particular success of this strategy is the controllable roughness of the surface on a conduit by exposing NCs to $3 \mathrm{D}$ printing, which not only increases the roughness for cell adhesion, but also manifests as a direct physical stimulus to cells (Fig. 1e). Furthermore, through exquisite fabrication, the microchannels of $\mathrm{NC}$ conduits effectively promote the cell alignment (Fig. 1f).

\section{Synthesis and characterization of NCs}

The robust composite $\mathrm{NCs}$ can be fabricated by employing GO as the substrate of the structure, as schematically illustrated in Fig. 2a. To prepare GO, the modified Hummers method was used. Similar to our previous work, a cationic surfactant (CTAB) was adsorbed onto the surface of synthesized GO via electrostatic interactions to form the micelle template. Under weakly basic conditions, the silica pore was constructed via the hydrolysis of tetraethyl orthosilicate and polymerization of styrene on the packed CTAB micelles on GO. Then, the particles were carbonized at high temperature under nitrogen to observe the NCs. TEM revealed that the mesoporous constructs were deposited on GO (Fig. 2b). With CTAB as the pore template, the pore size ranged from 3 to $6 \mathrm{~nm}$ in diameter, with an approximately random arrangement on the GO (Supplementary Fig. S2). Moreover, the chemical composition of graphite, GO, and 
NCs was estimated by XPS, and the analysis of the XPS patterns is displayed in Supplementary Fig. S3. A new, broad peak observed for GO at binding energies of $532 \mathrm{eV}$ was attributed to O1s (ref. ${ }^{37}$ ) and NCs at 154 and $174 \mathrm{eV}$ for the Si2p and Si2s components, respectively (Supplementary Fig. S3a). Furthermore, to provide insight regarding the surface-decorated functional groups, deconvoluted XPS spectra of C1s O1s and Si2p are shown in Supplementary Fig. S3b-d in the Supporting Information. In addition to displaying the peaks of GO, the $\mathrm{NCs}$ exhibited the formation of $\mathrm{C}-\mathrm{Si}$ and $\mathrm{C}-\mathrm{O}-\mathrm{Si}$ bonds in the structures. These results confirmed the successful exfoliation from graphite to GO by the Hummers method, and the attachment of carbon-containing silica onto the surface of the NCs. Furthermore, Raman spectroscopy was applied to evaluate the structure of graphite, GO, and NCs (Fig. 2c), using an excitation wavelength of $532 \mathrm{~nm}$. All samples were deposited in powder form on silicon wafers without using any solvent. The peak of the $G$ band is $\sim 1614 \mathrm{~cm}^{-1}$ (in-phase $\mathrm{sp}^{2}$ bond vibration), and the $\mathrm{D}$ band is $\sim 1360 \mathrm{~cm}^{-1}$ (disorder band of the graphene edge functional group). Two characteristic peaks were observed in all products. The relative peak ratio of G/D in graphene is higher than that in GO and NCs, which is probably caused by the low proportion of $\mathrm{sp}^{3}$ carbon centers (high content of $\mathrm{sp}^{2}$-hybridized $\mathrm{C}-\mathrm{C}$ bonds) ${ }^{38}$.

The synthesized GO with a mean size of $\sim 2 \mu \mathrm{m}$ was exfoliated from graphite, resulting in slow sedimentation of GO in water. After the formation of cookie structures on GO, the NCs were separated by centrifugation at 4000 r.p.m. to reduce their sizes, and the size distribution of the resulting NCs ranged from $\sim 300-550 \mathrm{~nm}$, as measured by DLS (Fig. 2d). Then, the BET method was utilized to investigate the pore structures through nitrogen absorption-desorption isotherms, which suggest that the surface area of the NCs was $\sim 550 \mathrm{~m}^{2} \mathrm{~g}^{-1}$; a pore size of $3.6 \mathrm{~nm}$ was determined by $\mathrm{BJH}$ analysis. The absorption-desorption curve exhibits typical Langmuir type IV characteristics with a hysteresis loop, which is the inherent property of mesoporous materials. Furthermore, the main hysteresis loop in the medium-pressure region indicates capillary condensation and multilayer adsorption in the mesopores. Compared to the NCs, both graphite and GO exhibited relatively low surface areas due to the nonporous structure (Fig. 2e and Supplementary Fig. S4).

\section{NFG release from NCs actuated by MF}

The magnetometry result of the NCs determined by a SQUID is given in Fig. 2f. The saturation magnetizations of the NCs were 0.001 and 0.4 e.m.u. $\mathrm{g}^{-1}$ at $300 \mathrm{~K}$ and $6 \mathrm{~K}$, respectively. The weak magnetic properties were induced by defects on the graphene. Through adsorption, the loading capacity of NGF in the NCs is $290 \mu \mathrm{g} \mathrm{g}^{-1} \mathrm{NC}$. The high loading efficiency mostly reflects an affinity between NGF and carbon surfaces, which NCs possess in abundance. Furthermore, to evaluate the effects of the MF on NGF release, the NGF-loaded NCs (NGF@NCs) were subjected to an MF for $15 \mathrm{~min}$ at a power of $3.2 \mathrm{~kW}$ $(1 \mathrm{MHz})$. A similar instrument developed by MagForce was approved for application in Europe to treat glioblastoma multiforme by local hyperthermia of iron oxide nanoparticles in brain tumors ${ }^{39}$. As shown in Fig. $2 \mathrm{~g}$, the amount of NGF released reached $\sim 45 \%$ after 15 min of MF treatment. Without MF treatment, the natural release of NGF was $\sim 14 \%$. The main reason for the triggered NGF release is the electrostatic repulsive force between the protein and NCs. According to Lenz's law, the direction of current in a conductor is induced by the external MF, which causes a MF that opposes the change that produced it. The magnetic flux causes the electromotive force in the conductive materials, and eddy currents are induced by the MF in closed loops within the stationary conductors ${ }^{40}$. As the NCs are a carbon-based conductive material, the MF potentially produces eddy currents in them. To investigate the MF effects on the $\mathrm{NCs}$, the NCs were exposed to an MF for $15 \mathrm{~min}$. After the MF treatment, the surface charge of the NCs estimated by a zeta potential analyzer changed to $-26.3 \mathrm{mV}$ from $-6.5 \mathrm{mV}$ due to the increased electrons on the particles. In addition, the NCs were slightly conductive, with a resistance of $1.64 \mathrm{k} \Omega \mathrm{cm}^{-1}$, which is similar to that of graphene and its derivatives ${ }^{41,42}$, and the eddy current could be induced in the bulk NCs (compact NCs formed by hydraulic press) by MF to light up an LED (Supplementary Fig. S5a). To evaluate the eddy current, NCs were placed in DMEM supplemented with 10\% FBS to mimic the physiological environment, and then subjected to an MF at various powers and distances with a frequency of $1 \mathrm{MHz}$. When subjected to an MF with a distance of $1 \mathrm{~cm}$, currents of $0.78,0.19$, and $0.1 \mu \mathrm{A}$ were measured at powers of $3.2,1.92$, and $0.32 \mathrm{~kW}$, respectively (Supplementary Fig. S5b). Once the distance between the MF and $\mathrm{NCs}$ was increased to $2 \mathrm{~cm}$, the induced currents were significantly decreased (Supplementary Fig. S5c).

\section{Electromagnetized NCs for PC12 cytotoxicity and differentiation}

The cytotoxicity of NCs with and without MF treatment was evaluated by incubating the NCs with PC12 cells, which were derived from a pheochromocytoma of a rat adrenal medulla line at different concentrations. Briefly, after coculturing the $\mathrm{NCs}$ and $\mathrm{PC} 12$ cells for $4 \mathrm{~h}$, the cells were subjected to 5 and 15 min of MF; then, cell viability was examined at $24 \mathrm{~h}$ posttreatment. To monitor the heat induced by the eddy current, as shown in Supplementary Fig. S6, at $120 \mu \mathrm{g} \mathrm{ml}^{-1} \mathrm{NCs}$, the temperature of the solution was observed to be slightly changed $\left(7^{\circ} \mathrm{C}\right)$ under 
$35^{\circ} \mathrm{C}$, and the viability of the cells with $5 \mathrm{~min}$ of $\mathrm{MF}$ treatment was $\sim 92 \%$, indicating that the short MF treatment did not exhibit an obvious influence on the cells. When the treatment time was increased to $15 \mathrm{~min}$, cell viability was maintained at $86 \%$, suggesting that the long treatment caused slight cell death due to energy generation. The cellular uptake of the NCs was examined by flow cytometry, as shown in Fig. $2 \mathrm{~h}$, which reveals significant fluorescence of the NCs after $0.5,1$, and $2 \mathrm{~h}$ of incubation.

To evaluate the MF effect on NGF@NCs in vitro, the PC12 cells were treated with an MF for $15 \mathrm{~min}$ at $1 \mathrm{~h}$ postincubation of the particles. Without the MF, NGF@NCs were observed on the cells (Fig. 2i), and the PC12 cells exhibited a short neurite length $(<15 \mu \mathrm{m})$ and a low differentiation percentage $(<4 \%)$. However, once the PC12 cells were treated with the MF, the average neurite length and differentiation percentage increased to $245 \mu \mathrm{m}$ and $18 \%$, respectively, indicating effective PC12 cell differentiation when combining the NGF@NCs and MF (Fig. 2j). To clarify the MF and NGF effects, both the PC12 cell neurite length and number of cells with neurites were studied under various conditions, as shown in Fig. 2k. With the assistance of the MF, the average neurite lengths of the PC12 cells reached 46 and $116 \mu \mathrm{m}$ for NCs and NGF@NCs, respectively. Generally, the neurite outgrowth of PC12 cells can be induced by NGF through activating the receptor tyrosine kinase, $G$ protein-coupled receptors, and heterotrimeric $G$ proteins. The results showed that the NCs were also able to induce PC12 cell differentiation without NGF; in addition, Fig. 2k (right panel) shows $8 \%$ of the cells with neurites under an MF. As documented in the literature, the electrical stimulation generated by locally charging objects is able to influence neuronal differentiation, biological processes, nerve regeneration processes, and neurite outgrowth through the upregulation of calcium signaling and phosphorylation of the cAMP-response element-binding pathway. Previous studies also documented that energy does not affect most cell types, but activates intracellular signaling to neurogenesis ${ }^{43}$.

As shown in Supplementary Fig. S7a, PC12 cells with and without attached NCs were placed together, and an MF was applied. PC12 cells attached to NCs exhibited clear enhancement of neurite outgrowth. Furthermore, to evaluate the effects of electromagnetized NCs on a biomaterial, NGF-NCs were coated on silk through layer-bylayer PLL/NC adsorption. As shown in Supplementary Fig. S7b, the surface of the silk displayed a rough morphology after NC coating, indicating a high density of NCs on the silk. Then, the PC12 cells were seeded on the substrate for $24 \mathrm{~h}$ of incubation, and the spherical morphology of PC12 cells was obtained because the cells were not differentiated with neurites (Supplementary Fig. S7c). While subjected to the MF for $15 \mathrm{~min}$ at a power of
$3.2 \mathrm{~kW}$, increased differentiation and neurite outgrowth were apparently observed on NC@silk (Supplementary Fig. S7d), and the synergistic effects of the NCs and MF were demonstrated on the PC12 cells (Supplementary Fig. S7e). This observation indicates that the electromagnetization of $\mathrm{NC}$ can be executed on a substrate and that cell fate can be manipulated, using an external physical energy source.

\section{D printing of $\mathrm{NC@C}$}

A light-curable bioink composed of 4-HBA, PU-EO-PO monomer, and Irgacure 819 (an initiator) could be polymerized by light with absorbance at a wavelength of $260 \mathrm{~nm}$ through 3D printing (Supplementary Fig. S8), and each curing layer in the DLP 3D printing process was $\sim 50 \mu \mathrm{m}$. 4-HBA has a primary hydroxyl group at the end of a long alkyl chain, providing stretchable and flexible characteristics. To enhance the water retention and protein permeability, the EO and $\mathrm{PO}$ segments served as hydrophilic and hydrophobic parts in the elastomer, which was capable of incorporating water-soluble molecules. With the loading of NCs or red dye, the resulting printed NC-loaded elastomer (NC@elastomer) exhibited solid morphology and excellent stability in various architectures (Fig. 3a). The image in Fig. 3b depicts a dogbone tensile specimen of the 3D printed elastomer. On the specimen, the appearance of the grid on the surface in the SEM image (the lower panel of Fig. 3b) resulted from the layer-by-layer printing and DLP light sources. The color and surface morphology of the sample would also be affected by adding NCs to the bioink, as shown in Fig. 3c, d. Once the concentration of NCs increased to $1 \%$, large amounts of NCs were exposed on the surface via the 3D printing process (Fig. 3e). This phenomenon reflects the local curing of DLP 3D printing, where the particles between light-emitting and unexposed areas were fixed on the resulting matrix surface (Supplementary Fig. S9). However, when traditional molding was used to prepare the composite, the particles preferred to be embedded in the gel. Thus, by using this printing technology, the surface roughness can be controlled, which has been demonstrated to improve cell adhesion ${ }^{44}$.

The precision of the 3D printed matrix was evaluated by Zen Desk software by comparing the resulting 3D printed matrix and the original CAD image (Supplementary Fig. S10). The precision was $87.55 \%$ and $82.5 \%$ for the elastomer and NC@elastomer, respectively. Furthermore, the resulting NC@elastomer displayed an elongation of $\sim 150 \%$ (Fig. 4a). While adding $10 \%$ of the PU-EO-PO monomer in the bioink, the elastic modulus could be $\sim 2.5$ times (Fig. 4b) because the aromatic urethane group of PU exhibits a strong intermolecular force. Moreover, by adding $1 \% \mathrm{NCs}$ to the elastomer, the elastic modulus was increased to $0.34 \mathrm{MPa}$ from $0.22 \mathrm{MPa}$ (Fig. 4c and 

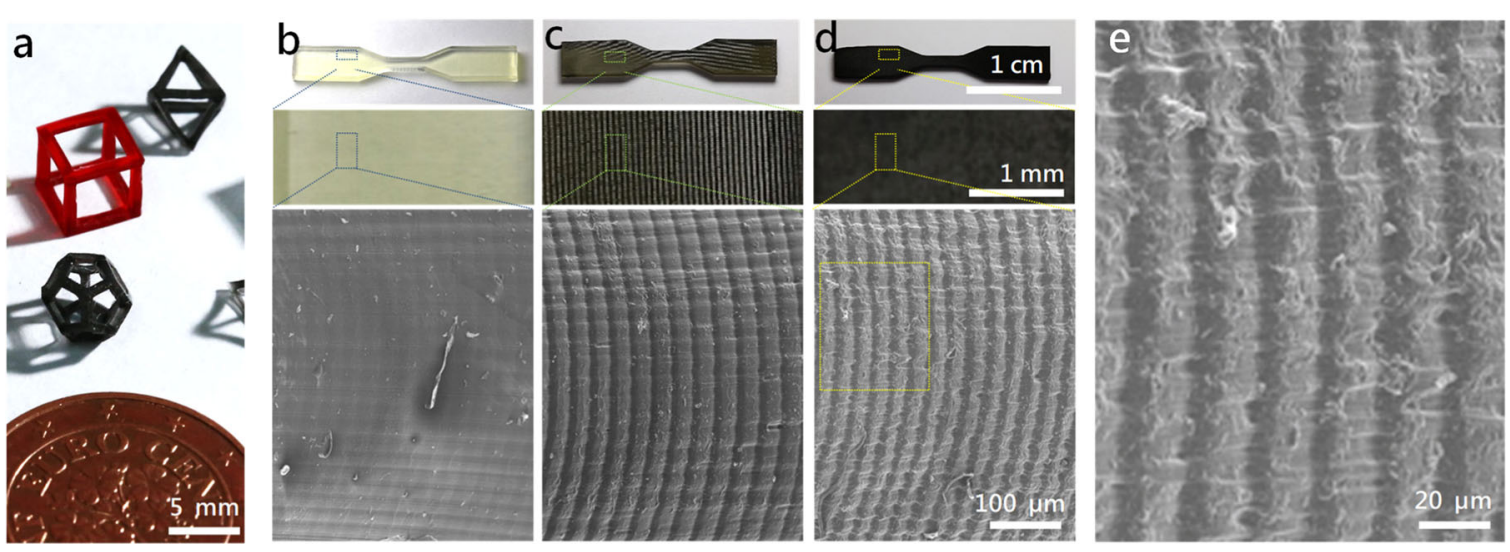

Fig. 3 Morphologies of 3D printed elastomers with NCs. a Various 3D printed geometry structures with and without NCs. Pictures and SEM images of 3D printed dog-bone specimens following ASTM-D638-14 (b) without NCs, (c) with $0.1 \%$ NCs and (d) with $1 \%$ NCs. e SEM image revealing the roughness of surface morphologies in the presence of NCs.

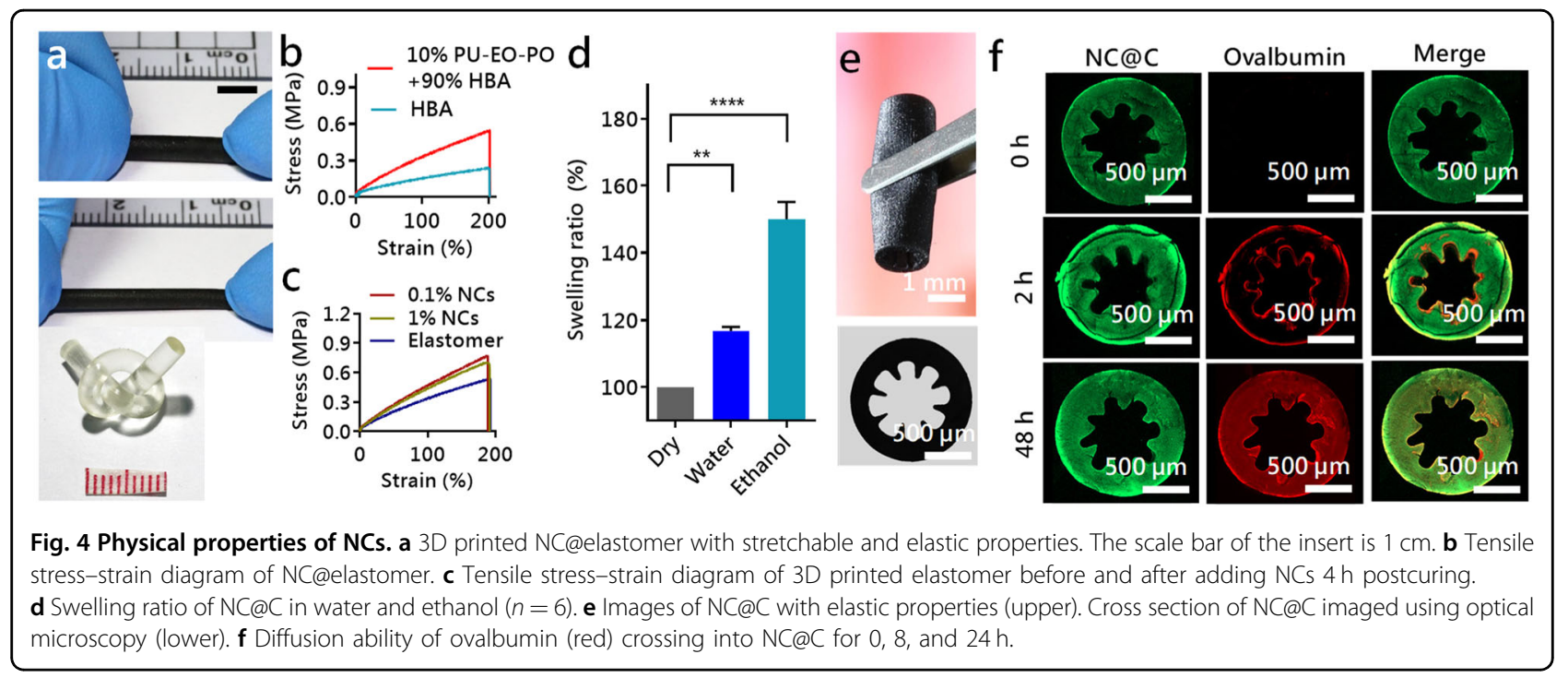

Supplementary Fig. S11). However, further addition of NCs to the bioink would lower the elasticity, so $1 \%$ NCs was used in the elastomer in the following drug/protein release, cell, and animal studies. Furthermore, to evaluate swelling behavior, the resulting NC@elastomer was placed in water and ethanol for $2 \mathrm{~h}$. As shown in Fig. 4d, with the assistance of a partial hydrophilic segment of PUEO-PO and NCs, the swelling ratio of the matrix was $\sim 15 \%$ and $46 \%$ in water and ethanol, respectively. The NC@C could still maintain the elasticity and microchannels after 2 weeks of immersion (Fig. 4e). After swelling, protein penetration was investigated by immersing Texas $\operatorname{Red}^{\mathrm{TM}}$-conjugated ovalbumin (ThermoFisher, USA) in the NC@C; then, the cross section of the conduit was examined by confocal laser scanning microscopy (CLSM, Zeiss LSM800, Germany). As shown in Fig. 4f, a significant fluorescence signal of ovalbumin was observed on the surface of the conduits after $2 \mathrm{~h}$ of diffusion, where the green fluorescence was caused by the autofluorescence of the conduit. After increasing the time to $48 \mathrm{~h}$, ovalbumin was detected in the whole conduit, and strong signals were also observed.

\section{NGF penetration and release from NC@C under MF treatment}

The protein penetration was potentially caused by the PU-EO-PO amphiphilic polymer chains and the NCs. Indeed, the $\mathrm{NC}$ amounts in the conduit reflected the protein-loading capacity of NC@C. As shown in Fig. 5a, the protein-loading capacity of $1 \% \mathrm{NC@C}$ was approximately three times greater than that of the conduit alone. However, $1 \% \mathrm{NCs}$ in the conduit did not obviously affect the loading capacity of small molecules when compared to that of the conduit alone, where doxorubicin (Dox) was 

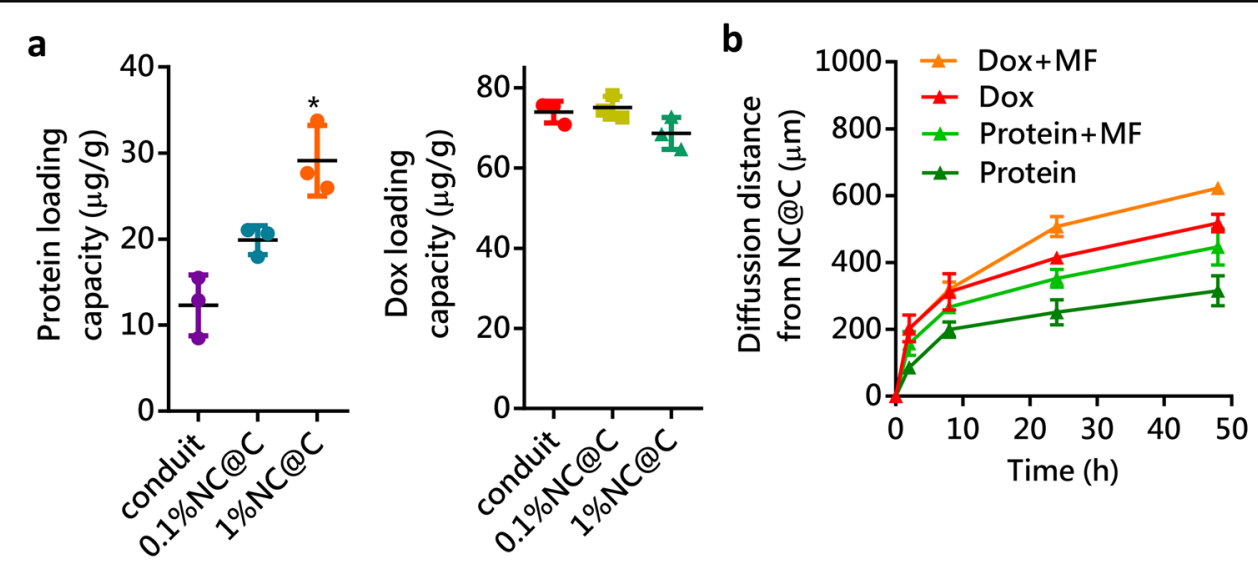

C

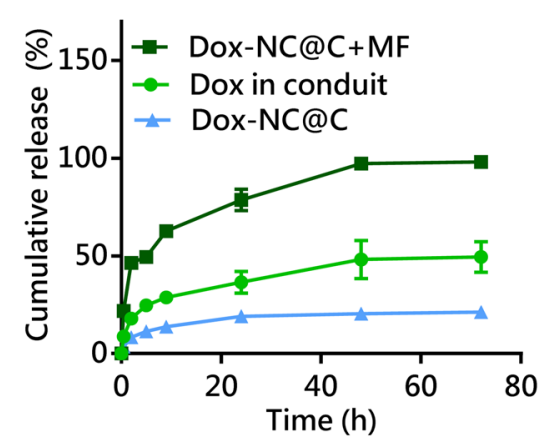

d

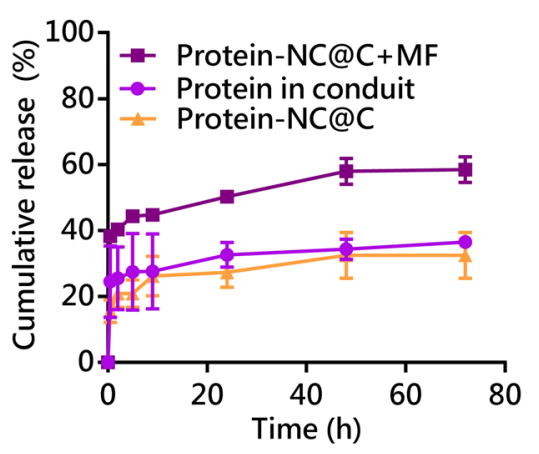

Fig. 5 Properties of capacity and release for NC@C. a Loading capacity of Dox and protein in the conduit, 0.1\%NC@C and 1\%NC@C ( $n=6$, mean \pm s.d., ${ }^{* *} p<0.01, t$-test). b Diffusion distance of Dox and protein from NC@C at various times $(n=6$, mean \pm s.d.). c Cumulative release of Dox from NC@C $(n=6$, mean \pm s.d.).d Cumulative release of protein from NC@C $(n=6)$. The MF was applied at a power of $3.2 \mathrm{~kW}$ and frequency of $1 \mathrm{MHz}$ for $15 \mathrm{~min}$.

used as a model drug. The mechanism was probably induced by the strong affinity between the carbon porous structure and the proteins, where the porous surface provided the binding sites to the proteins ${ }^{45}$. The external MF enhanced the penetration of Dox and ovalbumin into the NC@elastomer, which was monitored by CLSM from the top of the elastomer (Supplementary Fig. S12). The profiles of penetration depth under the influence of $15 \mathrm{~min}$ of MF treatment at the initial time point display several important features (Fig. 5b). (a) The penetration rate with an MF is much faster than that without an MF, as evident from the deep penetration of Dox and ovalbumin under MF treatment compared to that without. (b) The penetration rate of ovalbumin was slower than that of the small molecules, which could be understood in terms of the large molecular size of the protein. Similar triggered release results are displayed in Fig. $2 \mathrm{~g}$.

Since the MF is known to cause the local current to be mediated through the conductor of NCs, physical energy may enhance the chain flexibility of the elastomer, further aided by the affinity of the NCs to water, realizing the fast penetration of protein. Furthermore, the release patterns of Dox and NGF (a small and large model molecule, respectively) from NC@C under MF treatment were also investigated, as shown in Fig. 5c, d. For all the release profiles, there was an initial burst release, which manifested the rapid desorption of Dox and NGF from the surface of the conduit. Similar to the influence of the MF on penetration, the release rates were obviously increased while subjected to the MF due to the induced eddy current in the NCs. Although fast Dox and NGF release was obtained for the first hour to 3 days, the cumulative release of NGF reached only $60 \%$, whereas the Dox release was $\sim 100 \%$ due to the low diffusion rate of large molecules. Furthermore, Dox in conduits exhibited faster release than that in $\mathrm{NC@C}$, likely due to the strong affinity of NC for Dox. These results suggest that the short MF exposure caused a permanent conformation change that sustained the release of the cargo for a few days.

\section{Cell adhesion and PC12 differentiation on NC@C}

The in vitro cell attachment and compatibility of NC@C were determined using fibroblast cells (HIG-82), SCs, and N2a incubated for $24 \mathrm{~h}$. As shown in Fig. 6a, the three types 


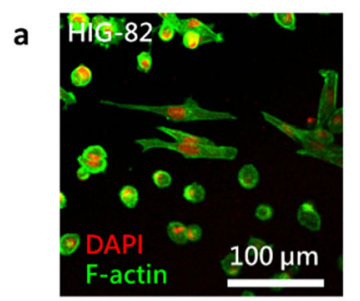

b

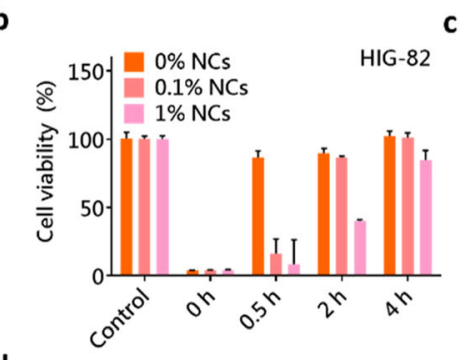

d
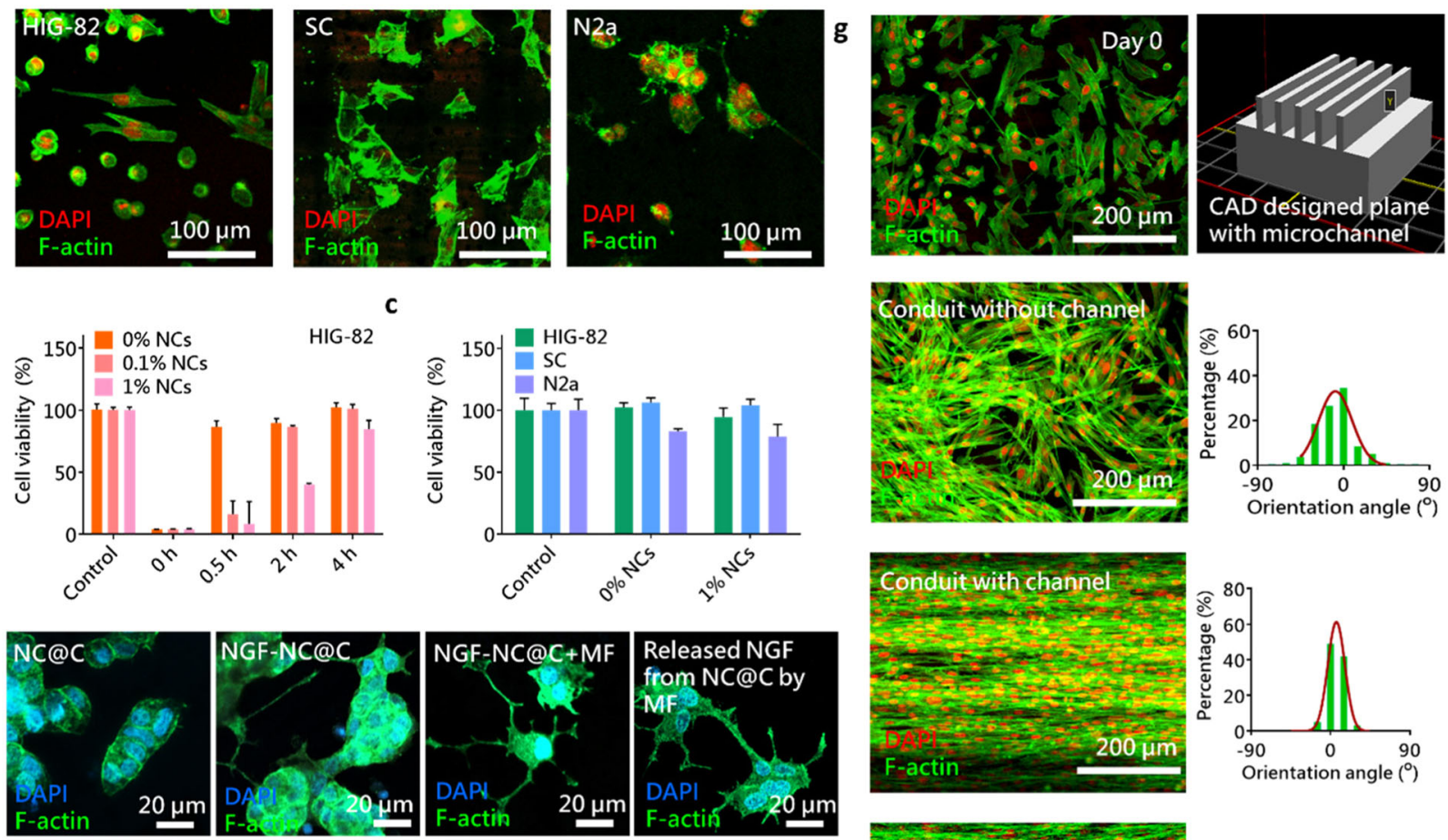

e
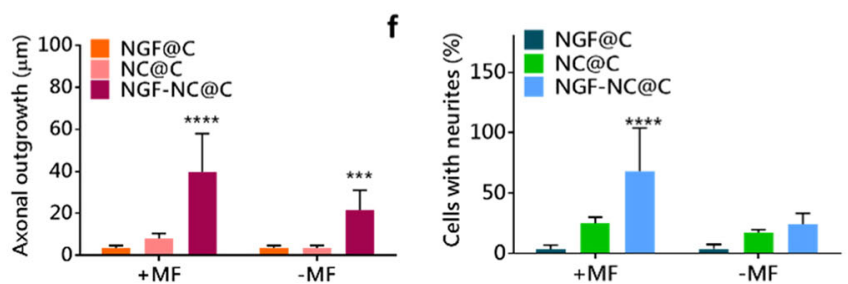

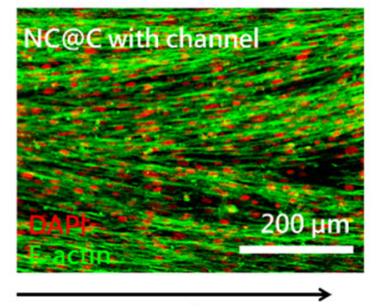

Orientation $\left(0^{\circ}\right)$

Fig. 6 In vitro cell adhesion on NC@C. a Morphologies of HIG-82, Schwann, and Neuro-2a cells on NC@C incubated for 1 day. DAPI stained the nucleus (red), and phalloidin stained F-actin (green). b Cell viability of HIG-82 cells incubated for $24 \mathrm{~h}$ with NC@C at various concentrations of NCs and postcuring times. c Cell viability of HIG-82, SC, and N2a cells incubated for $24 \mathrm{~h}$ with NC@C at various concentrations of NCs. d Fluorescence images of PC12 cells incubated with NGF-NC@C with and without MF treatment. e Quantitation of neurite length and $\mathbf{f}$ percentage of cells with neurites for PC12 cells in NGF@C, NC@C, and NGF-NC@C under MF. Statistical significance was determined using one-way ANOVA with Tukey's multiple comparison test $\left(n=6,{ }^{* * *} p<0.005,{ }^{* * *} p<0.001\right)$. g Fluorescence images of HIG-82 cells on NC@C with and without microchannels. The width and length of the microchannels are 0.7 and $10 \mathrm{~mm}$, respectively. Quantitation of axon orientation for HIG-82 cells (right column).

of cells effectively attached and developed on the surface, where DAPI stained the nucleus and phalloidin stained Factin. However, the conduit demonstrated weak cell adhesion. Compared to that on the cell culture dish, cell adhesion on the NC@C was $~ 80 \%$, but cell adhesion on the conduit alone was only $\sim 40 \%$, as shown in Supplementary Fig. S13. This observation is reminiscent of the effect of roughness through particle exposure, which is the basis of the $3 \mathrm{D}$ printing composite process. As expected, 1\% NCs increased the surface roughness of the conduits after the printing process, as shown in Supplementary Fig. S14. The profile roughness parameter ( $\mathrm{Ra}$ ) increased from 2.93 to 36.32 when adding $1 \% \mathrm{NCs}$ to the bioinks during 3D printing. Having demonstrated cell adhesion, we followed ISO 10993-5 (tests for in vitro cytotoxicity) to determine the cell viability of the 3D printed materials before removal of unreacted agents. As shown in Fig. 6b, higher cell viability of the 3D printed materials was observed after $4 \mathrm{~h}$ postcuring in HIG-82 cells. The results indicated that polymers containing residual initiators and monomers would lead to considerable toxicity ${ }^{46}$. Therefore, the postcuring and removal of unreacted agents in the polymer by ethanol were apparently critical in the application of photocured NC@C. Figure $6 \mathrm{c}$ also reveals that the NCs slightly decreased the cell viability of the conduits, suggesting the low toxicity of the latter. Therefore, before NGF loading and animal studies were carried out, these agents were removed to mitigate toxicity.

For effective nerve outgrowth, differentiation should be triggered by an external physical cue and growth factors. 
For NC@C, we investigated the percentage of PC12 cells differentiated with neurites and the length of axonal outgrowth induced by NGF and MF (Supplementary Fig. S15a). Similar to a previous study, NCs treated with the MF impacted $\beta$-III-tubulin and the neuron-specific cytoskeletal protein in PC12 cells through the synergistic effect of NGF and electrical stimulation (Fig. 6d). As expected, the fluorescence images of PC12 cells with MF stimulation exhibited longer neurite extensions than those cells without treatment. To estimate the NGF (protein) activity after MF treatment, NGF was loaded on NCs in advance and released by applying an MF. The released NGF was then added to PC12 cells. As the results showed that the released NGF was still able to induce PC12 cell differentiation, indicating the activity of NGF (Fig. 6d). Statistical analysis of neurite length and neurite number over various treatments is also demonstrated in Fig. 6e, f. In the NGF-NC@C group, the average neurite length reached $40 \mu \mathrm{m}$, and $>60 \%$ of the PC12 cells were differentiated after 15 min of MF treatment; for the PC12 cells without MF treatment, the average neurite length reached only $22 \mu \mathrm{m}$, and $31 \%$ of the cells were differentiated. The results confirmed the significant enhancement in the differentiation of $\mathrm{PC} 12$ cells under MF treatment through electromagnetic stimulation of the cells and intense NGF release. To further evaluate the effects of $\mathrm{NC}$ exposure on the conduits, the NCs were embedded in a gel to model PC12 cell differentiation. As shown in Supplementary Fig. S15b, c, while subjected to the MF, the NGF-NC-embedded gel could induce cell differentiation by triggering NGF release. However, compared to that for the exposed $\mathrm{NC}$ on the conduit, the neurite length or percentage of cells with neurites for the NGF-NC-embedded gel was decreased, indicating that the amplification of $\mathrm{PC} 12$ cells was stimulated by directly contacting the NCs to cells. Therefore, 3D printing to expose NCs on the conduits can significantly enhance cell differentiation. To ascertain the effects of the microchannels on cell orientation, HIG-82 cells were cultured on $3 \mathrm{D}$ printed conduits containing microgrooves with and without NCs. As shown in Fig. 6g, the cells on the conduit without channels had a random orientation. However, in the groups of conduits with channels, the HIG-82 cells were highly aligned after 4 days of incubation. This orientation was also quantified by a Gaussian distribution (oriented at a $0^{\circ}$ basis angle), as shown in the right column of Fig. $6 \mathrm{~g}$, suggesting the effective improvement of in vitro cell orientation via these channels. Compared with the conduit with the channel (without NC), NC@C with the channel exhibited less orientation, which was potentially attributed to the surface roughness, facilitating cell adhesion $^{47,48}$. To clarify the differences in the generated eddy currents in vitro and in vivo, the NCs were placed in DMEM supplemented with $10 \%$ FBS to mimic the cell culture conditions and biological tissue (animal muscle), respectively. The generated currents in vitro and in vivo were $\sim 28$ and $\sim 38 \mu \mathrm{A}$, respectively (Supplementary Fig. S16). The differences may be attributed to the ions in the microenvironments.

\section{Functional recovery of a regenerated sciatic nerve in vivo}

To implement the spatial distribution of NGF for the promotion of axon outgrowth, 3D printed NC@C loaded with NGF was implanted after a peripheral nerve was transected $10 \mathrm{~mm}$ from the bifurcation point of the sciatic nerve in the popliteal fossa of SD rats. In the animal studies, five groups, including autograft, NC@C, NGFNC@C, and NGF-NC@C+MF, were implanted in vivo in the sciatic nerve transection, where identical amounts of NGF $\left(29 \mu \mathrm{g} \mathrm{g}^{-1}\right.$ conduit) were applied in the NGF-loaded groups (Fig. 7a). The distance between the MF and the incision at the posterior of the left hind was maintained at $1 \mathrm{~cm}$ (Supplementary Fig. S17). The MF treatment was carried out for $15 \mathrm{~min}$ at a power of $3.2 \mathrm{~kW}$ and frequency of $1 \mathrm{MHz}$. The toe spreading of an injured hind paw and the SFI were measured 30 days postimplantation, which is an atypical walking footprint analysis of toe spreading. The SFI is 0 for healthy animals and -100 for animals with a transected sciatic nerve. After 1 month of treatment, a significantly wide toe spreading of the injured hind paw was observed in the NGF-NC@C+MF group, indicating excellent axonal regeneration (Fig. 7b, c), where $15 \mathrm{~min}$ of MF was locally applied at 4 days postimplantation at a power of $3.2 \mathrm{~kW}(1 \mathrm{MHz})$. After analysis, we observed that the SFI value of the NGF-NC@C $+\mathrm{MF}$ group was higher than those in the other groups. Furthermore, once sciatic nerve injury occurs without treatment, it causes atrophy of the gastrocnemius muscle (decreases in muscle weight and muscle fiber size), leading to a decreased weight ratio of the wet gastrocnemius muscles between the left and right sides. However, in the NGF-NC@C + MF group, the weight ratio of the gastrocnemius muscles was close to that in the autograft group, suggesting excellent recovery of the gastrocnemius muscles (Fig. 7d).

For peripheral nerve regeneration, the morphologies of atrophied muscle were used to estimate limb recovery. At 1 month postimplantation, the gastrocnemius muscles were harvested and stained by hematoxylin and eosin, as shown in Supplementary Fig. S18. Then, the diameter and distribution of the muscle fibers were calculated (Fig. 7e). The atrophied muscle in the conduit alone group demonstrated obvious degradation of muscles. Even though the weight ratio of the gastrocnemius muscles of the NGF-NC@C group was close to that of the autograft, weak recovery was observed in the muscle fibers, i.e., small fiber diameter. However, combining the MF treatment, the diameter of the muscle fibers was regained after 


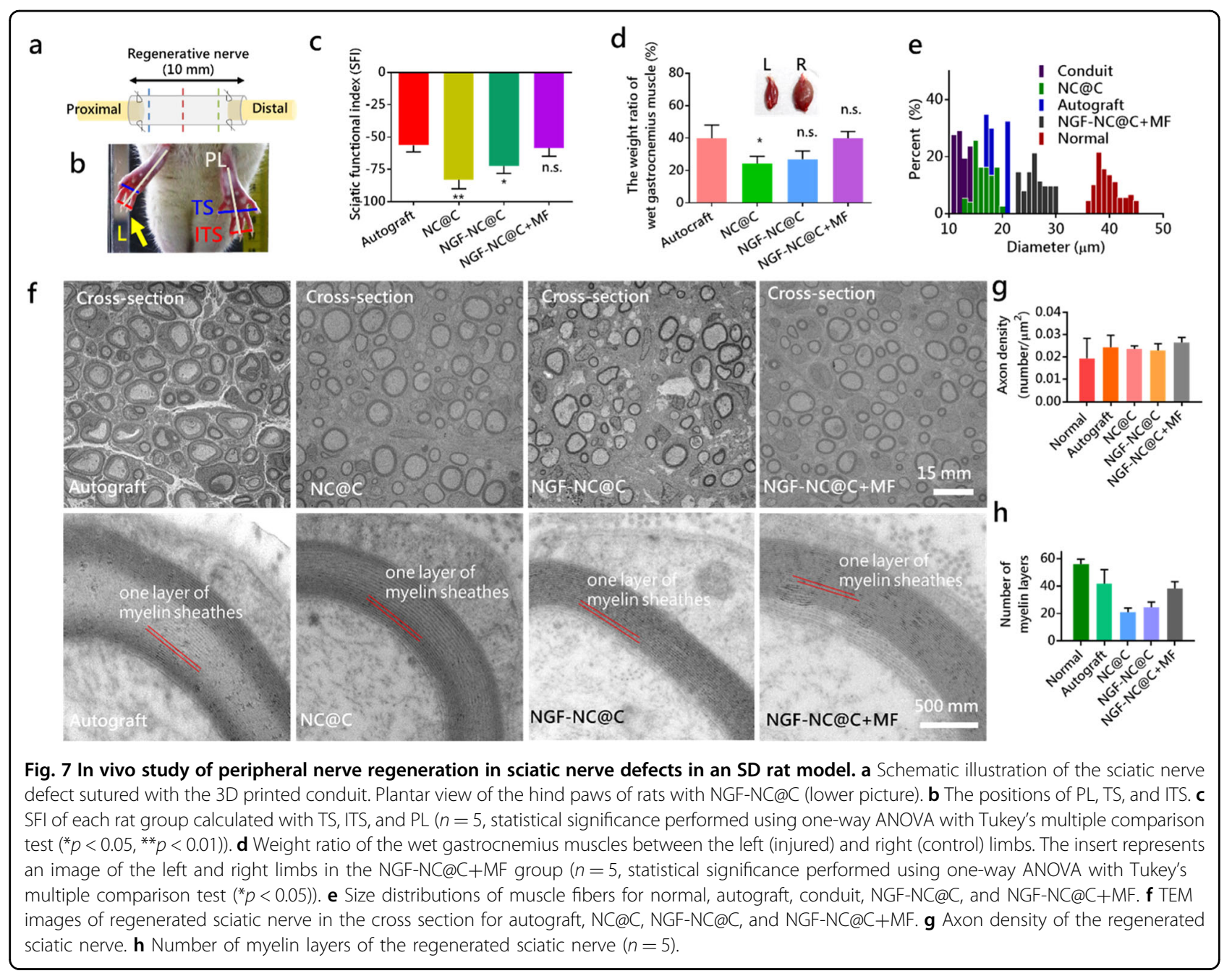

nerve reinnervation and was close to that in the autograft (gold standard in nerve gap repair), as observed in the NGF-NC@C+MF group.

\section{Regenerated myelinated nerve fibers}

The myelinization process of SCs is a key factor in axonal regeneration after PNI because the developing nervous system is guided by a myelin sheath. Furthermore, distal SCs undergo atrophy due to the loss of axonal contact for a prolonged period, resulting in an inhibitory extracellular environment for nerve regeneration. Generally, stronger distal axons and myelination lead to better functional recovery for the injured nerve. As shown in Fig. $7 \mathrm{f}$, the cross section of distal regenerative nerves evaluated by TEM at 1 month postimplantation suggests that the lamination of myelin sheathes of the regenerated nerve fibers in NGF-NC@C+MF was successful compared to that of the gold standard (autograft). However, in the NC@C and NGF-NC@C groups, the number of layers of myelin sheaths was smaller. After myelination analysis, the axon densities in each group were similar, but only NGF-NC@C+MF exhibited a similar number of myelin layers as that of the autograft (Fig. 7g, h). These findings indicated that NGF-NC@C+MF improved the diameter of the regenerative axon by controlling biocues and magnetoelectric stimulation.

To understand the growth and orientation of regenerated axons, the whole regenerated nerve from the proximal to distal section in the conduit was harvested, and stained with B-III-tubulin and DAPI to track the regenerated axons and cell nuclei, respectively. Figure 8 displays a large area of CLSM images of immunohistochemically stained sciatic nerve defects from various treatments at 1 month postimplantation. Several conclusions could be drawn from these results. First, although the autograft group had a thick nerve, curled and random fiber-like morphologies of regenerated axons were observed because the direct surgical suture led to the mismatch of axons. Second, NGF-NC@C+MF exhibited a thicker nerve bundle than NGF-NC@C and NC@C, indicating 

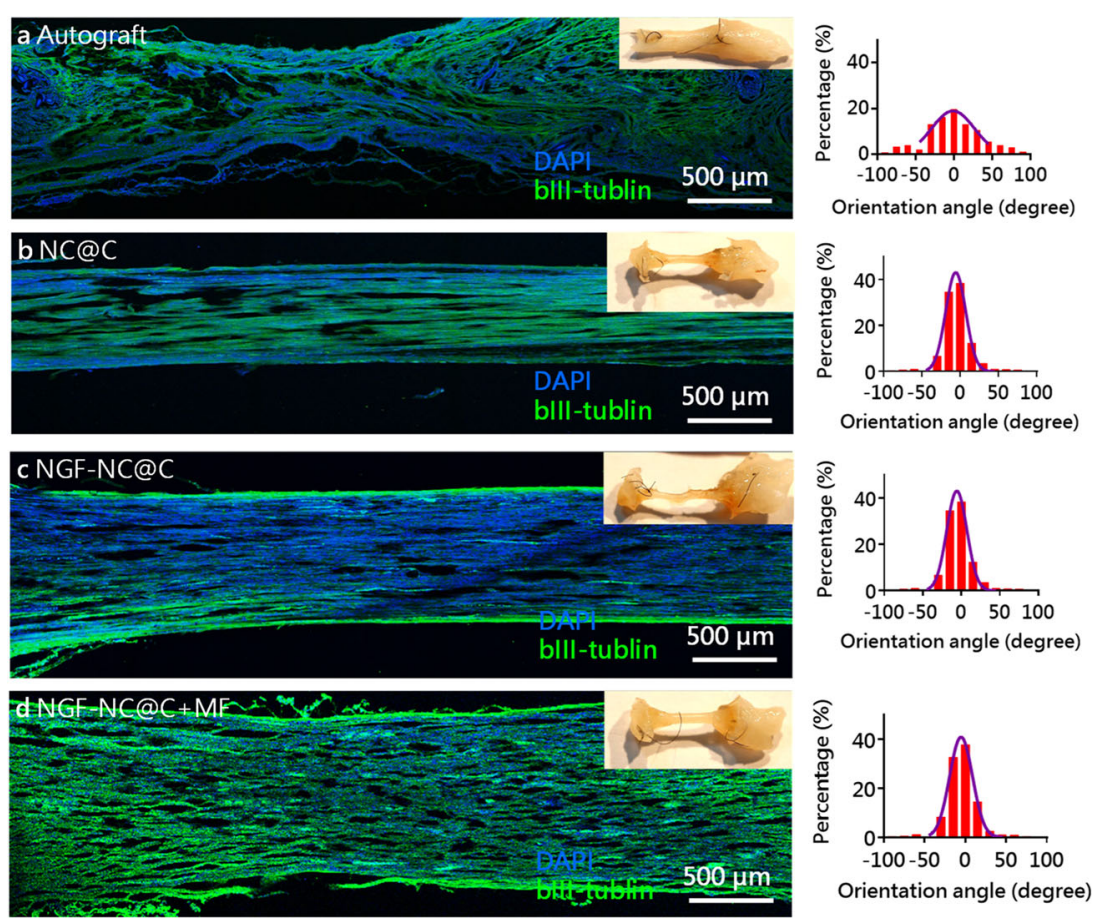

Fig. 8 Immunohistochemistry images of peripheral nerve regeneration. Sciatic nerve defects harvested from a autograft, b NC@C, c NGF-NC@C, and $\mathbf{d}$ NGF-NC@C+MF $(n=5)$. The inserted images show the optical images, and the bar charts show the orientation analysis. Direct axonal outgrowth followed the microchannels of the conduit. Green represents $\beta$-III-tubulin (axon), and blue represents nuclei. The orientation is from proximal to distal $(n=5)$.

that nerve growth was promoted by magnetoelectric stimulation and NGF release (inset picture). Third, a small distribution of axon orientation was detected in the conduit groups. This observation confirmed the effective regeneration of the MF treatment (i.e., magnetoelectric stimulation induced nerve differentiation mediated by NCs in the conduit, and NGF release enhanced cell growth). In addition, judging from the limb muscle weights and functions, the nerve recovery effects in NGF$\mathrm{NC@C+MF} \mathrm{appear} \mathrm{to} \mathrm{be} \mathrm{excellent.} \mathrm{Electrophysiological}$ analysis includes nerve conduction velocity (NCV) compound muscle action potential (CMAP) in Supplementary Fig. S19a, which is a classical electrophysiological method with diagnostic and prognostic value for evaluating $\mathrm{PNI}^{49,50}$. NCV and CMAP are highly influenced by axons and myelin, which are correlated with the maturation of regenerative nerve fibers ${ }^{17,51}$. A normal nerve was found to exhibit an NCV of $47.5 \mathrm{~m} \mathrm{~s}^{-1}$ and a CMAP of $46.2 \mathrm{mV}$. At 1 month postsurgery, the NGF-NC@C+MF group exhibited an NCV of $44.7 \mathrm{~m} \mathrm{~s}^{-1}$ and a CMAP of $44.9 \mathrm{mV}$, both of which were higher than those of the NGF-NC@C group (Supplementary Fig. S19b, c).

To estimate the immune response for a long period, the autograft nerve and NC@C were harvested and compared after 2 months of surgery, where the numbers and distribution of macrophages were stained by CD68 antibody for tracking purposes (Supplementary Fig. S20a, b). The CLSM images revealed that there was no significant difference in the numbers of macrophages between the normal and NC@C groups, indicating low inflammation in the tissue. Furthermore, the weight changes of mice for autograft, conduit, NC@C, and NC@C+MF displayed almost identical patterns due to the biocompatibility of the implanted materials (Supplementary Fig. S20c). Next, six indices of liver as well as kidney functions, including albumin, alkaline phosphatase, alanine aminotransferase, aspartate aminotransferase, creatinine, and total bilirubin, and two strong predictors of all-cause mortality includingserum C-reactive protein and plasma D-dimers, were also evaluated after 60 days postimplantation of highdensity polyene (control group), conduit, and NC@C (Supplementary Fig. S21). The differences between these groups were relatively small, implying the low toxicity of various treatments and safety of the materials. On the other hand, no severe immune response or tissue damage was observed once NC@C was implanted in muscle tissue, following the ISO 10993-6 test (tests for local effects after implantation, Supplementary Fig. S22). These results are consistent with previous studies exhibiting tolerable bioresponses for a long time.

In summary, we have developed a 3D printing method to prepare an $\mathrm{NC}$-exposed conduit with stretchable and 
biocompatible characteristics. The $\mathrm{NC}$, a nanocomposite composed of reduced GO, mesoporous silica, and a thin carbon layer, possesses superb attributes of magnetoelectric conversion, protein adsorption, and cell stimulation. After integrating the NCs into a bioink, the printed NC@C was able to carry large amounts of NGF with high efficiency and exhibited molecule/protein permeability. On-demand NGF release and cell stimulus were achieved in vitro using a high magnetoelectric treatment to effectively induce cell proliferation and differentiation, regaining the diameter of animal muscle fibers, as well as improving layers of myelin sheathes in vivo and directing the axon orientation. This printed NC@C with exposed particles, excellent mechanical properties, controllable NGF release, and magnetoelectric stimulus is expected to introduce a new approach for the regeneration of nerves and other tissues.

\section{Acknowledgements}

This work was financially supported by the Ministry of Science and Technology of the Republic of China, Taiwan under contracts MOST 109-2636-E-007-014 and MOST 108-2636-E-007-001, and by NTHU (107Q2512E1) in Taiwan.

\section{Author details \\ ${ }^{1}$ Department of Biomedical Engineering and Environmental Sciences, National Tsing Hua University, Hsinchu 300, Taiwan. ${ }^{2}$ Center for Biomedical Engineering, Chang Gung University, Taoyuan City 333, Taiwan. ${ }^{3}$ Department of Biomedical Engineering, National Yang-Ming University, Taipei City 112, Taiwan. \\ ${ }^{4}$ Department of Materials Science and Engineering, National Chiao Tung University, Hsinchu 300, Taiwan. ${ }^{5}$ Frontier Research Centre on Fundamental and Applied Sciences of Matters, National Tsing Hua University, Hsinchu 310, Taiwan. ${ }^{6}$ School of Dentistry, College of Dental Medicine, Kaohsiung Medical University, Kaohsiung 807, Taiwan. ${ }^{7}$ Graduate Institute of Biomedical Science, China Medical University, Taichung 404, Taiwan}

\section{Conflict of interest}

The authors declare that they have no conflict of interest.

\section{Publisher's note}

Springer Nature remains neutral with regard to jurisdictional claims in published maps and institutional affiliations.

Supplementary information is available for this paper at https://doi.org/ 10.1038/541427-020-00244-1.

Received: 31 December 2019 Revised: 27 May 2020 Accepted: 3 July 2020. Published online: 11 September 2020

\section{References}

1. Fu, H. et al. Morphable 3D mesostructures and microelectronic devices by multistable buckling mechanics. Nat. Mater. 17, 268-276 (2018).

2. Yu, K., Xin, A., Du, H., Li, Y. \& Wang, Q. Additive manufacturing of self-healing elastomers. NPG Asia Mater. 11, 7 (2019).

3. Liu, G., Zhao, Y., Wu, G. \& Lu, J. Origami and 4D printing of elastomer-derived ceramic structures. Sci. Adv. 4, eaat0641 (2018).

4. Kim, Y., Parada, G. A., Liu, S. \& Zhao, X. Ferromagnetic soft continuum robots. Sci. Robot. 4, eaax7329 (2019).

5. Cui, H. et al. Three-dimensional printing of piezoelectric materials with designed anisotropy and directional response. Nat. Mater. 18, 234-241 (2019).

6. Trenfield, S. J., Awad, A., Goyanes, A., Gaisford, S. \& Basit, A. W. 3D Printing pharmaceuticals: drug development to frontline care. Trends Pharmacol. Sci. 39, 440-451 (2018).
7. Wang, X. et al. A 3D-printed scaffold with $\mathrm{MoS}_{2}$ nanosheets for tumor therapy and tissue regeneration. NPG Asia Mater. 9, e376 (2017).

8. Liu, X. et al. 3D printing of living responsive materials and devices. Adv. Mater. 30, 1704821 (2018)

9. Schaffner, M., Rühs, P. A., Coulter, F., Kilcher, S. \& Studart, A. R. 3D printing of bacteria into functional complex materials. Sci. Adv. 3, eaao6804 (2017).

10. Yu, F. et al. Real-time manipulation of intestinal peristalsis by entericencapsulated magnetic nanoparticles \& wearable 3D-printed devices. NPG Asia Mater. 11, 33 (2019).

11. Truby, R. L. et al. Soft somatosensitive actuators via embedded 3D printing. Adv. Mater. 30, e1706383 (2018).

12. Liang, K., Carmone, S., Brambilla, D. \& Leroux, J. C. 3D printing of a wearable personalized oral delivery device: a first-in-human study. Sci. Adv. 4, eaat2544 (2018).

13. Zhao, Y. et al. Biomimetic composite scaffolds to manipulate stem cells for aiding rheumatoid arthritis management. Adv. Funct. Mater. 29, 1807860 (2019).

14. Johnson, B. N. et al. 3D printed anatomical nerve regeneration pathways. Adv. Funct. Mater. 25, 6205-6217 (2015).

15. Höke, A. Mechanisms of disease: what factors limit the success of peripheral nerve regeneration in humans? Nat. Clin. Pract. Neurol. 2, 448-454 (2006).

16. Shahriari, D. et al. Scalable fabrication of porous microchannel nerve guidance scaffolds with complex ceometries. Adv. Mater. 31, 1902021 (2019).

17. Hsu, R. S. et al. Adaptable microporous hydrogels of propagating NGFgradient by injectable building blocks for accelerated axonal outgrowth. Adv. Sci. 6, 1900520 (2019).

18. Van Huizen, A. V. et al. Weak magnetic fields alter stem cell-mediated growth. Sci. Adv. 5, eaau7201 (2019).

19. Gratwicke, J. et al. Bilateral deep brain stimulation of the nucleus basalis of meynert for parkinson disease dementia: A randomized clinical trial. JAMA Neurol. 75, 169-178 (2018)

20. $\mathrm{Wu}, \mathrm{C} . \mathrm{H}$. et al. Trojan-horse nanotube on-command intracellular drug delivery. Nano Lett. 12, 5475-5480 (2012).

21. Lim, K. T. et al. Pulsed-electromagnetic-field-assisted reduced graphene oxide substrates for multidifferentiation of human mesenchymal stem cells. Adv. Healthc. Mater. 5, 2069-2079 (2016).

22. Yoo, J. et al. Electromagnetized gold nanoparticles mediate direct lineage reprogramming into induced dopamine neurons in vivo for Parkinson's disease therapy. Nat. Nanotechnol. 12, 1006-1014 (2017).

23. Ng, S., Noor, N. \& Zheng, Z. Graphene-based two-dimensional Janus materials. NPG Asia Mater. 10, 217-237 (2018).

24. Lee, J. H. et al. Nondestructive real-time monitoring of enhanced stem cell differentiation using a graphene-Au hybrid nanoelectrode array. Adv. Mater. 30, e1802762 (2018).

25. Gao, T. et al. Transferable, transparent and functional polymer@graphene 2D objects. NPG Asia Mater. 6, e130 (2014).

26. Choi, S., Kim, S. \& Kim, I. Ultrafast optical reduction of graphene oxide sheets on colorless polyimide film for wearable chemical sensors. NPG Asia Mater. 8 , e315 (2016).

27. Zhao, G. et al. Reduced graphene oxide functionalized nanofibrous silk fibroin matrices for engineering excitable tissues. NPG Asia Mater. 10, 982-994 (2018).

28. Yang, $\mathrm{H}$. et al. Printing hydrogels and elastomers in arbitrary sequence with strong adhesion. Adv. Funct. Mater. 29, 1901721 (2019).

29. Hung, K. C., Tseng, C. S., Dai, L. G. \& Hsu, S. H. Water-based polyurethane 3D printed scaffolds with controlled release function for customized cartilage tissue engineering. Biomaterials 83, 156-168 (2016).

30. Farokhi, M., Mottaghitalab, F., Fatahi, Y., Khademhosseini, A. \& Kaplan, D. L. Overview of silk fibroin use in wound dressings. Trends Biotechnol. 36, 907-922 (2018).

31. Qian, Y. et al. An integrated multi-layer 3D-fabrication of PDA/RGD coated graphene loaded PCL nanoscaffold for peripheral nerve restoration. Nat Commun. 9, 323 (2018).

32. Niu, Y. et al. Scaffolds from block polyurethanes based on poly( $\varepsilon$-caprolactone) $(\mathrm{PCL})$ and poly(ethylene glycol) (PEG) for peripheral nerve regeneration. Biomaterials 35, 4266-4277 (2014).

33. Huang, G. et al. Functional and biomimetic materials for engineering of the three-dimensional cell microenvironment. Chem. Rev. 117, 12764-12850 (2017).

34. Qing, H. et al. Heterostructured silk-nanofiber-reduced graphene oxide composite scaffold for SH-SY5Y cell alignment and differentiation. ACS Appl. Mater. Interfaces 10, 39228-39237 (2018). 
35. Hummers, W. S. Jr \& Offeman, R. E. Preparation of graphitic oxide. J. Am. Chem. Soc. 80, 1339 (1985).

36. Su, Y. L. et al. The penetrated delivery of drug and energy to tumors by lipographene nanosponges for photolytic therapy. ACS Nano 10, 9420-9433 (2016).

37. Kudin, K. N. et al. Raman spectra of graphite oxide and functionalized graphene sheets. Nano Lett. 8, 36-40 (2008).

38. Zarrin, H., Higgins, D., Jun, Y., Chen, Z. \& Fowler, M. Functionalized graphene oxide nanocomposite membrane for low humidity and high temperature proton exchange membrane fuel cells. J. Phys. Chem. C 115, 20774-20781 (2011).

39. Chiu-Lam, A. \& Rinaldi, C. Nanoscale thermal phenomena in the vicinity of magnetic nanoparticles in alternating magnetic fields. Adv. Funct. Mater. 26, 3933 (2016)

40. Wang, P. et al. Assembly-induced thermogenesis of gold nanoparticles in the presence of alternating magnetic field for controllable drug release of hydrogel. Adv. Mater. 28, 10801-10808 (2016).

41. Pang, S., Hernandez, Y., Feng, X. \& Müllen, K. Graphene as transparent electrode material for organic electronics. Adv. Mater. 23, 2779-2795 (2011).

42. Bonaccorso, F., Sun, Z., Hasan, T. \& Ferrari, A. C. Graphene photonics and optoelectronics. Nat. Photonics 4, 611-622 (2010).

43. Lynch, K. J., Skalli, O. \& Sabri, F. Growing neural PC-12 cell on crosslinked silica aerogels increases neurite extension in the presence of an electric field. J. Funct. Biomater. 9, 30 (2018).
44. Li, Y. et al. Antibody-modified reduced graphene oxide films with extreme sensitivity to circulating tumor cells. Adv. Mater. 27, 6848-6854 (2015).

45. Yang, Y. \& Li, J. Lipid. protein and poly(NIPAM) coated mesoporous silica nanoparticles for biomedical applications. Adv. Colloid Interface Sci. 207, 155-163 (2014).

46. Chan, H. N. et al. Direct, one-step molding of 3D-printed structures for convenient fabrication of truly 3D PDMS microfluidic chips. Microfluid Nanofluid 19, 9-18 (2015)

47. Shuai, Y., Mao, C. \& Yang, M. Protein nanofibril assemblies templated by graphene oxide nanosheets accelerate early cell adhesion and induce osteogenic differentiation of human mesenchymal stem cells. ACS Appl. Mater. Interfaces 10, 31988-31997 (2018).

48. Li, Y. et al. Antibody-modified reduced graphene oxide films with extreme sensitivity to circulating tumor cells. Adv. Mater. 2015, 6848-6854 (2015).

49. Raimondo, T. M. et al. Combined delivery of VEGF and IGF-1 promotes functional innervation inmice and improves muscle transplantation in rabbits. Biomaterials 216, 119246 (2019).

50. Wang, G. W., Yang, H., Wu, W. F., Zhang, P. \& Wang, J. Y. Design and optimization of a biodegradable porous zein conduit using microtubes as a guide for rat sciatic nerve defect repair. Biomaterials 131, 145-159 (2017).

51. Das, S. et al. In vivo studies of silk based gold nano-composite conduits forfunctional peripheral nerve regeneration. Biomaterials 62, 66-75 (2015). 